\title{
Évolution récente de la maintenance du matériel roulant à la SNCF (1972-2002)
}

Recent evolutions of rolling stock maintenance at the SNCF (1972-2002)

Alain Bernheim

\section{OpenEdition}

\section{Journals}

Édition électronique

URL : https://journals.openedition.org/rhcf/1758

DOI : $10.4000 /$ rhcf. 1758

\section{Éditeur}

Rails \& histoire

\section{Édition imprimée}

Date de publication : 1 décembre 2003

Pagination : 129-135

ISBN : 0996-9403

ISSN : 0996-9403

\section{Référence électronique}

Alain Bernheim, «Évolution récente de la maintenance du matériel roulant à la SNCF (1972-2002) », Revue d'histoire des chemins de fer [En ligne], 28-29 | 2003, mis en ligne le 19 décembre 2014, consulté le 22 avril 2022. URL : http://journals.openedition.org/rhcf/1758 ; DOI : https://doi.org/10.4000/rhcf. 1758

Ce document a été généré automatiquement le 22 avril 2022

Tous droits réservés 


\section{Évolution récente de la maintenance du matériel roulant à la SNCF (1972-2002)}

Recent evolutions of rolling stock maintenance at the SNCF (1972-2002)

Alain Bernheim

1 La SNCF a connu une grande mutation technologique lorsque, après la reconstruction du réseau détruit par la Seconde Guerre mondiale, elle fit muter la traction vapeur vers la traction électrique et thermique. Ceci nécessita une importante reconversion de son personnel. Après cette étape technologique, la SNCF engagea une réforme de son management en constituant 25 régions en 1972, celles-ci étant dotées de pouvoirs renforcés en matière de gestion du personnel et en prenant contact avec le monde institutionnel régional.

2 En 1972, on dénombre 163 établissements du Matériel, non compris 44 dépôts relais (tableau 1, en annexe) trente années plus tard, en 2002, les 25 régions SNCF sont devenues 23 et l'on ne dénombre plus que 59 établissements du Matériel (tableau 2, en

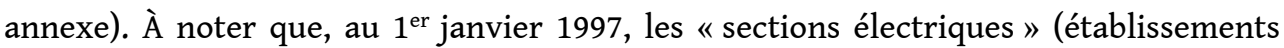
chargés des équipements d'alimentation des lignes électrifiées) ont été transférées dans les services de l'Infrastructure.

3 Les effectifs du domaine Matériel ont évolué de 43000 agents environ (1972) vers 24000 agents environ (2002).

4 Sur cette même période, le trafic SNCF exprimé en unités-kilomètres ( $u k=v k+t k$, voyageurs-kilomètres + tonnes-kilomètres) a évolué de 111 G.uk (milliards d'unitéskilomètres) en 1972 à 124 G.uk en 2002 (prévision).

5 Ces résultats parlent d'eux-mêmes pour caractériser le renforcement de la productivité de ce secteur.

$6 \quad$ Au plan des idées, les évolutions ont été importantes. 


\section{2 - La logique industrielle}

7 Cette logique s'est donné les objectifs suivants :

8 - qualité

9 - moindre coût

10 - climat de travail.

11 Elles s'appuyait sur des principes :

12 - responsabilité ) «tout passe par l'homme »

13 - compétence )

14 Elle s'est dotée de moyens :

15 - formation

16 - spécialisation

17 - stabilité

18 Une vigoureuse politique de maintenance s'est affichée, travaillant sur les deux axes techniques et humains.

19 Le principe de spécialisation a été largement décliné :

20 - spécialisation des établissements,

21 - spécialisation des équipes, des sections et des ateliers,

22 - spécialisation des agents.

23 L'élaboration des règles de maintenance a été minutieusement décentralisée et adaptée à chaque cas. La répartition des missions par spécialité, par activité et par organe a fait l'objet de nombreuses concertations, cette organisation industrielle a été un succès et a permis de maîtriser l'outil ferroviaire moderne et de dégager de nouvelles performances.

24 Le succès n'a été obtenu que par une animation forte du domaine « $\mathrm{M}$ », grâce à la volonté de ses dirigeants et à la mise en œuvre de cellules d'appui baptisées à l'époque "section organisation». Une rigoureuse gestion des ressources humaines a permis d'imprégner tous les esprits de ces principes et de ces objectifs, le corps social a finalement largement adhéré à ces idéaux.

\section{5 - La démarche qualité}

25 L'expérience des cercles de qualité introduits dans le monde industriel européen a inspiré une prise de conscience analogue au sein du monde ferroviaire.

Pour stimuler la recherche du « bien faire », la notion de « client interne » a été mise en exergue et a abouti au fait que chaque acteur du système ne travaille que pour servir son ou (ses clients).

27 Après l'identification du client, le débat porte sur le «Produit », la démarche pouvant se schématiser de la façon suivante :

28 - phase conception : clients et fournisseurs négocient la définition du produit.

29 - Phase de production : le fournisseur réalise le produit convenu pour le client, selon le cahier des charges convenu, au prix convenu et dans les délais convenus. 

des charges. l'on pouvait se demander : qui dirige quoi? ferme. résultats à la direction de l'entreprise.

La qualité se définit par : réaliser un produit conforme à celui décrit dans les cahiers

La pratique de cette démarche n'a pas soulevé d'opposition, les seules difficultés éventuelles se situant dans l'identification des clients.

\section{0 - La SNCF accentue la décentralisation des pouvoirs internes}

Les dirigeants de l'époque pensaient améliorer l'efficacité de l'entreprise en "forçant » de nouvelles décentralisations, démarche qui revient à jouer le pouvoir des directeurs de Région contre celui des domaines nationaux. À titre d'exemple, les «Réseaux Matériel " sont supprimés, de même que les sections organisation, la politique «Ressources humaines » du domaine Matériel est mise à mal ; les principes forts qui avaient permis la modernisation de l'outil ferroviaire se sont affadis, à un point tel que

\section{6 et au-delà - Nouvelle croissance du trafic ferroviaire par la politique de volume}

La SNCF a été fortement secouée par les mouvements sociaux de fin 1995. La nouvelle équipe dirigeante, à partir de 1996, oriente une politique de volume, au management

La balkanisation à laquelle a conduit la politique des années 1990 est patente ; l'outil ferroviaire est très intégré ; il a besoin d'un pilotage fort. Les domaines techniques sont invités à reprendre fermement en main les affaires, de manière à présenter de vrais

5 S'il est constaté que les principes de 1972 ont toute leur valeur, les moyens pour les remettre en œuvre ont été fortement réduits dans l'intervalle, d'où la difficulté des équipes dirigeantes actuelles; à cela s'ajoute l'évolution récente.

\section{0/2002 - La logique du service au client}

Les principes du Matériel n'ont de valeur que si la performance économique est au rendez-vous. Avec une croissance du trafic très forte, plus forte que prévu, les exploitants veulent engager de plus en plus de matériel. L'objectif numéro un assigné au domaine $\mathrm{M}$ devient celui de la disponibilité.

Les organisations du terrain doivent évoluer, les régimes horaires de travail aussi (augmentation des services décalés, augmentation du travail les week-ends).

Au plan « philosophie », le constat suivant pourrait être énoncé :

- la mise en place de la «nouvelle organisation» de 1972 a privilégié l'ergonomie de l'homme au travail en prévoyant dans toute la mesure du possible le travail de jour et sur la semaine, même si cela consommait du parc de matériel. 
40 - La politique de volume d'aujourd'hui nécessite que l'homme soit plus souple dans ses conditions de travail de façon que le capital rapporte plus (meilleures rotations du matériel roulant).

41 La traduction concrète se retrouve dans le projet industriel de la SNCF, décliné en «programmes prioritaires»:

42 - augmenter la disponibilité des locomotives,

43 - augmenter la disponibilité des voitures GL (grandes lignes) dans les centres de maintenance,

44 - augmenter la disponibilité des TGV.

45 L'efficacité du domaine Matériel, reconnue par l'entreprise, lui vaut d'augmenter son périmètre d'action. Le projet "Produit Train» est engagé dans tous les grands chantiers de maintenance des rames classiques à voyageurs.

46 L'ancien partage des tâches sur ces sites, entre "Transport » et « Matériel », laissera la place à un établissement de production unique « $\mathrm{M}$ », l'objectif étant de livrer à l'exploitant un produit complet (maintenance et activités d'exploitation connexes); cette évolution avait été expérimentée avec succès lors de la constitution des centres de maintenance TGV, où ceux-ci ont d'emblée gérés eux-mêmes leurs manœuvres internes.

47 Un autre aspect de la politique de volume est de nécessiter pour l'appareil de production $\mathrm{M}$ un accroissement de productivité inusité à ce jour. II y a là un véritable défi, nécessitant un partage des objectifs par tous.

\section{Conclusion}

Le renouveau du transport ferroviaire s'appuie sur les performances du matériel roulant (performances techniques et économiques). Si des états d'âmes pouvaient troubler les esprits dans un certain passé, l'heure est plus que jamais à l'action pour permettre de gagner les nouvelles batailles commerciales et économiques, les acquis d'aujourd'hui constituant un formidable capital gageant la réussite demandée.

MTA : département des matériels automoteurs ; MTC : département des locomotives, de wagons et des convois ; MTT : département des TGV et voitures ; MTT-V : division des voitures.

\section{ANNEXES}

Annexe 
Tableau 1. Les établissements Matériel de la SNCF en 1972

\begin{tabular}{|c|c|c|c|c|c|c|c|c|}
\hline Régions SNCF & Atelier MM & Atelier MR & Dépôt titulaire & Dépôt relais & Entretien V. W. & Смт & SNE & MG \\
\hline PARIS-EST & & 1 & 1 & & 3 & & 1 & \\
\hline REIMS & 1 & & 2 & 1 & 2 & 1 & 1 & \\
\hline METZ & & 1 & 2 & 1 & 4 & 2 & 1 & \\
\hline NANCY & & & 1 & 2 & 2 & 1 & 1 & \\
\hline STRASBOURG & 1 & & 1 & 2 & 3 & 1 & 1 & \\
\hline PARIS-NORD & & 1 & 2 & 2 & 3 & 1 & 1 & \\
\hline AMIENS & & 1 & 1 & 2 & 3 & 2 & & 1 \\
\hline LILLE & 1 & & 2 & 5 & 7 & 1 & 1 & 1 \\
\hline PARIS-ST-LAZARE & 1 & & 2 & 1 & 1 & & 1 & \\
\hline ROUEN & 1 & & 2 & 2 & 2 & 3 & & \\
\hline PARIS-MONTPARNASSE & 1 & & 1 & 2 & 2 & & & \\
\hline NANTES & & & 2 & 1 & 1 & 1 & & \\
\hline RENNES & & 1 & 1 & 2 & 1 & 2 & & \\
\hline PARIS-SUD-OUEST & 1 & & 2 & & 2 & & 2 & \\
\hline TOURS & & & 1 & 1 & 1 & 1 & 2 & 1 \\
\hline LE MANS & & 1 & 2 & 2 & 1 & & 2 & \\
\hline BORDEAUX & 1 & 1 & 1 & 1 & 1 & 2 & 2 & \\
\hline TOULOUSE & & & 2 & 1 & 2 & & 3 & \\
\hline PARIS-SUD-EST & & 1 & 1 & 2 & 2 & 1 & 1 & \\
\hline DIJON & & 1 & 1 & 3 & 1 & & 1 & \\
\hline CLERMONT-FERRAND & 1 & & 1 & 3 & 2 & & & \\
\hline CHAMBERY & & & 2 & 2 & 1 & 1 & 1 & \\
\hline LYON & 1 & 1 & 3 & 2 & 2 & & 1 & \\
\hline MARSEILLE & & 1 & 2 & 3 & 3 & & 1 & \\
\hline MONTPELLIER & & 1 & 2 & 1 & 1 & & 1 & \\
\hline 25 & 10 & 12 & 40 & (44) & 53 & 19 & 26 & 3 \\
\hline
\end{tabular}

CMT : centre Matériel et Traction ; SNE : section électrique ; MG : magasins généraux. Total : 163 établissements (207 avec les dépôts relais) - 1982 : « nationalisation » de l'IP Cadoux qui est devenu l'EIMM de St-Pierre-des-Corps

Tableau 2. Les établissements du domaine Matériel de la SNCF et leur « département pilote » à la direction du Matériel en 2002

\begin{tabular}{|c|c|c|}
\hline Région & Établissement & Pilote \\
\hline \multirow{3}{*}{ PARIS-EST } & EMT de Paris-Est & MTA \\
\hline & EMM de l'Ourcq & MTT-V \\
\hline & EIMM de Romilly & MTT-V \\
\hline \multirow{6}{*}{ METZ-NANCY } & EMM Châlon-en-Champagne & MTC \\
\hline & EIMM Épernay & MTC \\
\hline & EMT Chalindrey & MTC \\
\hline & EMT Nord-Lorraine & MTC \\
\hline & EMM Metz & MTC \\
\hline & EIMM de Montigny & MTC \\
\hline \multirow{3}{*}{ STRASBOURG } & EMT de Strasbourg-Hausbergen & MTC \\
\hline & EMM de Strasbourg & MTC \\
\hline & EIMM de Bischeim & MTT \\
\hline \multirow{2}{*}{ PARIS-NORD } & EMR de Paris-Nord & MTA \\
\hline & EIMM du Landy & MTT \\
\hline \multirow{2}{*}{ AMIENS } & EMT d'Amiens-Longueau & MTC \\
\hline & EIMM de Tergnier-Longueau & MTC \\
\hline \multirow{2}{*}{ LILLE } & EMM de Lille & MTC \\
\hline & EIMM d'Hellemmes & MTT \\
\hline \multirow{2}{*}{ PARIS-ST-LAZARE } & EMT d'Achères-Clichy & MTA \\
\hline & EMT Paris-St-Lazare & MTA \\
\hline \multirow{3}{*}{ ROUEN } & ET de Caen & MTA \\
\hline & EMM de Normandie & MTC \\
\hline & EIMM de Quatre-Mares & MTC \\
\hline \multirow{2}{*}{ RENNES } & EMT de Bretagne & MTC \\
\hline & EIMM de Rennes & MTA \\
\hline \multirow{2}{*}{ NANTES } & EMM de Nantes & MTT-V \\
\hline & EIMM du Mans & MTA \\
\hline \multirow{5}{*}{ PARIS-RIVE-GAUCHE } & EMT de Paris-Sud-Ouest & MTA \\
\hline & EMT de Paris-Atlantique & MTA \\
\hline & EMM de Trappes & MTT-V \\
\hline & EIMM de Paris-Massena & MTT-V \\
\hline & EIM-TGV de Chatillon & MTT \\
\hline \multirow{2}{*}{ TOURS } & EMT de Tours-St-Pierre & MTA \\
\hline & EIMM de St-Pierre-des-Corps & MTA \\
\hline LIMOGES & EMT Limousin & MTA \\
\hline
\end{tabular}

MTA : département des matériels automoteurs; MTC : département des locomotives, de wagons et des convois ; MTT : département des TGV et voitures ; MTT-V : division des voitures. 
(tableau 2. suite)

\begin{tabular}{|c|c|c|}
\hline Région & Établissement & Pilote \\
\hline \multirow{4}{*}{ BORDEAUX } & EMT de Sud-Aquitaine & MTT-V \\
\hline & EMM de Bordeaux & MTT-V \\
\hline & EIMM de Saintes & MTA \\
\hline & El du Périgord & MTT-V \\
\hline \multirow{2}{*}{ TOULOUSE } & EMT de Toulouse & MTC \\
\hline & EMM de Toulouse & MTT-V \\
\hline \multirow{3}{*}{ PARIS-SUD-EST } & EMT de Villeneuve & MTA \\
\hline & EMM de Villeneuve-Prairie & MTT-V \\
\hline & EIM-TGV de Paris-Sud-Est & MTT \\
\hline \multirow{2}{*}{ DIJON } & EMT de Dijon & MTC \\
\hline & EMM de Dijon-Perrigny & MTC \\
\hline \multirow{4}{*}{ LYON } & EMT de Venissieux & MTA \\
\hline & EMT de Lyon-Vaise & MTA \\
\hline & EMM de Lyon & MTT-V \\
\hline & EIMM d'Oullins & MTC \\
\hline \multirow{2}{*}{ CLERMONT-FERRAND } & EMT d'Auvergne & MTA \\
\hline & EMT de Nevers & MTA \\
\hline \multirow{2}{*}{ CHAMBERY } & Établissement Mont-Blanc & MTA \\
\hline & EMR de Chambéry & MTT-V \\
\hline \multirow{4}{*}{ MARSEILLE } & EMT de Nice & MTT-V \\
\hline & EMT de Marseille & MTC \\
\hline & EMT d'Avignon & MTC \\
\hline & EMM de Miramas & MTC \\
\hline MONTPELLIER & Languedoc-Roussillon & MTC \\
\hline
\end{tabular}

\section{RÉSUMÉS}

Cette contribution traite de l'organisation et de la politique générale qui ont structuré la maintenance $d u$ matériel roulant à la SNCF entre 1972 et 2002. Après la reconversion technologique exigée après la Seconde Guerre mondiale par la traction thermique et électrique, le domaine fit face à la fois à la réduction de ses moyens et à l'accroissement considérable de la demande, sous-tendue par un trafic en forte augmentation. L'article distingue ainsi cinq grandes phases dans la conception du métier au cours de la période étudiée.

D’abord, à partir de 1972 s'établit la « logique industrielle », héritière directe du passé. 1985 initia ensuite l'adoption d'un mode de pensée extérieur, la « démarche qualité ». Puis l'introduction en 1990 d'une décentralisation accrue déstructura partiellement le domaine au nom de l'efficacité. Ce premier stade difficile fut suivi, à partir de 1996, par une politique de "volume" accompagnant la croissance du trafic. Enfin, l'année 2002 introduisit la «logique du service au client » visant à assurer une disponibilité maximum du matériel (jusqu'à penser le "produit train » livré à l'exploitation), quitte à faire évoluer les pratiques et l'organisation historiques du travail des agents du domaine Matériel.

This contribution deals with the organization and general policy which have structured maintenance of rolling stock in the SNCF between 1972 and 2002. After the technological conversion required after World War II by thermal and electrical traction, a new challenge emerged in the area, who faced both the reduction of its ressources and the considerable increase in demand (underpinned by a sharp increase in traffic). One must therefore distinguish five major phases in the design of the activity during the period. 
From 1972 first, is implemented the "industrial logic", direct heir of the past. Then it is 1985 and the adoption of a way of thinking coming from outside, the "quality approach". In 1990, the introduction of increased decentralisation partially undermined the domain in the name of efficiency. This difficult phase was followed, from 1996, by a policy of "volume" accompanying the growth of traffic. Finally, the year 2002 introduced the "logic of customer service" to ensure maximum availability of the equipment (up to think the "product train", delivered to the Operation), even to the price of an evolution of historical practices and organization of work of the « Matériel » agents.

\section{INDEX}

Mots-clés : dépôt, France, organisation du travail, maintenance

Keywords : depot, France, maintenance, organization of labor

\section{AUTEUR}

\section{ALAIN BERNHEIM}

Rédacteur en chef de la Revue générale des chemins de fer, directeur honoraire de la SNCF (Matériel et Traction) 